\title{
Multi-Step Ubiquitin Decoding Mechanism for Proteasomal Degradation
}

\author{
Hikaru Tsuchiya, Akinori Endo and Yasushi Saeki * \\ Protein Metabolism Project, Tokyo Metropolitan Institute of Medical Science, Tokyo 156-8506, Japan; \\ tsuchiya-hk@igakuken.or.jp (H.T.); endo-ak@igakuken.or.jp (A.E.) \\ * Correspondence: saeki-ys@igakuken.or.jp; Tel.: +81-3-6834-2329
}

Received: 8 June 2020; Accepted: 22 June 2020; Published: 23 June 2020

\begin{abstract}
The 26S proteasome is a 2.5-MDa protease complex responsible for the selective and ATP-dependent degradation of ubiquitylated proteins in eukaryotic cells. Proteasome-mediated protein degradation accounts for $\sim 70 \%$ of all cellular proteolysis under basal conditions, and thereby any dysfunction can lead to drastic changes in cell homeostasis. A major function of ubiquitylation is to target proteins for proteasomal degradation. Accompanied by deciphering the structural diversity of ubiquitin chains with eight linkages and chain lengths, the ubiquitin code for proteasomal degradation has been expanding beyond the best-characterized Lys48-linked ubiquitin chains. Whereas polyubiquitylated proteins can be directly recognized by the proteasome, in several cases, these proteins need to be extracted or segregated by the conserved ATPases associated with diverse cellular activities (AAA)-family ATPase p97/valosin-containing protein (VCP) complex and escorted to the proteasome by ubiquitin-like (UBL)-ubiquitin associated (UBA) proteins; these are called substrate-shuttling factors. Furthermore, proteasomes are highly mobile and are appropriately spatiotemporally regulated in response to different cellular environments and stresses. In this review, we highlight an emerging key link between p97, shuttling factors, and proteasome for efficient proteasomal degradation. We also present evidence that proteasome-containing nuclear foci form by liquid-liquid phase separation under acute hyperosmotic stress.
\end{abstract}

Keywords: proteasome; protein degradation; ubiquitin; proteasomal ubiquitin receptors; $\mathrm{p} 97 / \mathrm{VCP}$; liquid-liquid phase separation

\section{Introduction}

The ubiquitin-proteasome system is the primary degradation system in eukaryotic cells. The $26 \mathrm{~S}$ proteasome is a huge enzyme complex composed of the 20S core particle (CP; also known as 20S proteasome) and one or two $19 \mathrm{~S}$ regulatory particles (RPs). There are 33 distinct proteins, with a total of 66 subunits, which constitute $26 \mathrm{~S}$ proteasome. The $\mathrm{CP}$ has a barrel-shaped structure that possesses two sets of three proteolytic active sites inside the cavity. The RP recognizes the polyubiquitin chains via intrinsic ubiquitin receptors (Rpn10, Rpn13, and Rpn1), unfolds the substrate proteins, and translocates them into the catalytic $\mathrm{CP}$ [1]. Besides the RP, the proteasome has alternative proteasome activators, such as PA2 $\alpha \alpha / \beta$ (PSME1/2), PA28 $\gamma$ (PSME3), and PA200 (PSME4), which are involved in ubiquitin-independent protein degradation (reviewed by Kish-Trier et al.) [2]. Through the degradation of substrates, the proteasome plays an essential role in most cellular functions, such as cell cycle progression, transcription, signal transduction, and protein homeostasis [3]. In addition to the constitutive subunits, the proteasome has multiple transiently-associated proteins called proteasome-interacting proteins. Among them, substrate shuttling factors, ubiquitin ligases, and deubiquitylating enzymes modulate proteasome functions. It is now clear that ubiquitin-selective AAA-ATPase p97 and substrate shuttling factors like RAD23 and UBQLNs act to select and deliver ubiquitylated substrates upstream of the 
proteasome [4]. In this review, we describe the recent progress in our research on factors upstream of the proteasome, including substrate shuttling factors and p97, the selectivity of the ubiquitin chain for proteasomal degradation, and recently identified nuclear proteasome droplets that are formed under the hyperosmotic stress triggered by multivalent interactions between RAD23B and ubiquitylated proteins to be degraded. These findings underline the multi-step regulation of proteasome responding to the cellular environment.

\section{Ubiquitin-Binding Proteins Related to the Proteasome: Proteasomal Ubiquitin Receptors, UBL-UBA Proteins, and p97}

\subsection{Proteasomal Ubiquitin Receptors (Rpn10, Rpn13, and Rpn1)}

In proteasomal degradation, the recognition of ubiquitin chains is one of the most crucial steps. The proteasome has three defined intrinsic ubiquitin receptors, namely Rpn10, Rpn13, and Rpn1, that recognize ubiquitylated substrates on the proteasome. These ubiquitin receptors can also bind ubiquitin-like (UBL) domains and are therefore also known as UBL domain receptors (Figure 1).

a

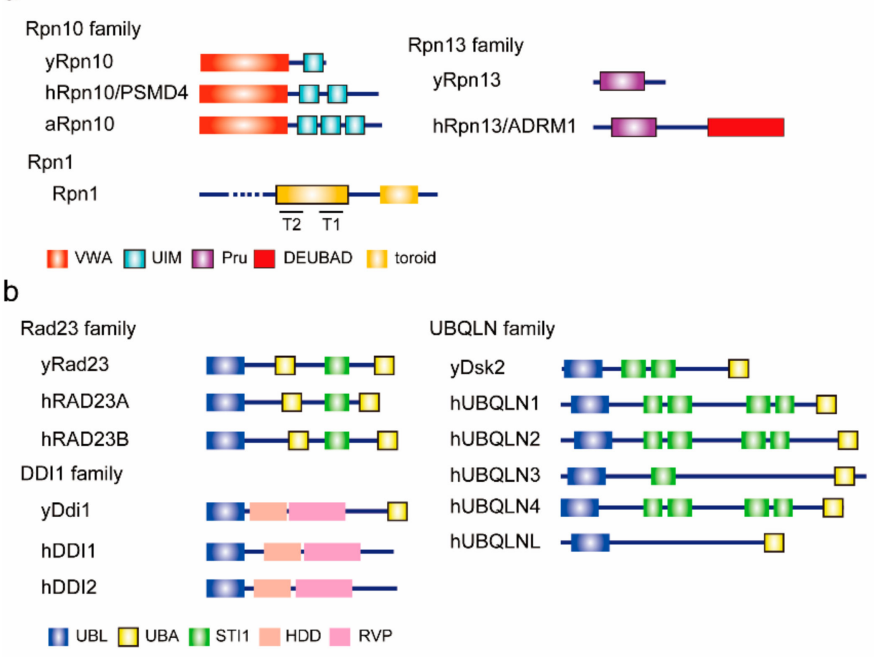

C

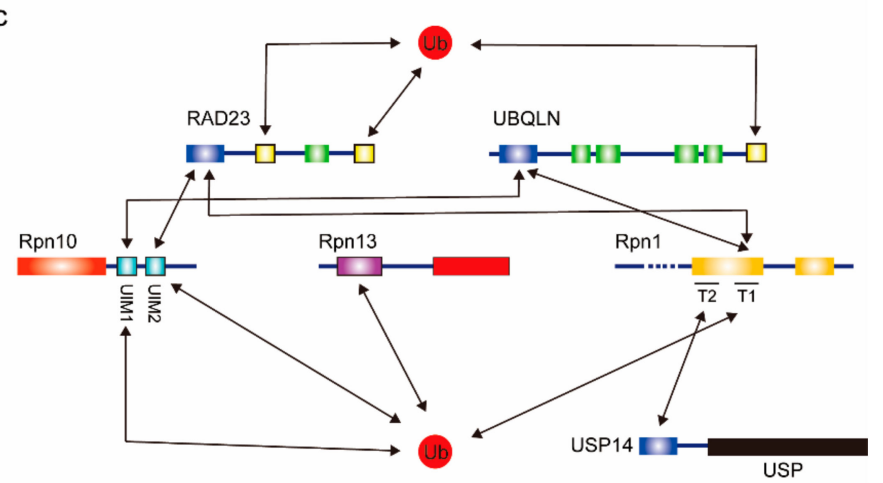

Figure 1. Proteasomal ubiquitin receptors. (a) Domain architectures of intrinsic proteasomal ubiquitin receptors. Known ubiquitin and ubiquitin-like (UBL) domain-binding domains are indicated by thick-bordered boxes. The prefix " $\mathrm{h}$ " indicates the human protein; " $\mathrm{y}$ " indicates the yeast protein; "a" indicates the arabidopsis protein. VWA, von Willebrand factor A. UIM, ubiquitin-interacting motif. Pru, Pleckstrin-like receptor for ubiquitin. DEUBAD, deubiquitinase adaptor. (b) Domain architectures of extrinsic ubiquitin receptors. UBL, ubiquitin-like. UBA, ubiquitin associated. HDD, helical domain of Ddi1. RVP, predicted retroviral protease-like. (c) The interaction map for ubiquitin and ubiquitin receptors. Known interactions are indicated by arrows. USP, ubiquitin-specific protease. 
Rpn10 (also known as S5a/PSMD4) is composed of an N-terminal von Willebrand factor A (VWA) domain and C-terminal ubiquitin-interacting motif (UIM) domains. The VWA domain interacts with the proteasome and the UIM domain binds to ubiquitin chains. The UIM domain consists of a single alpha helix. Yeast Rpn10 has one UIM domain, but human and plant Rpn10 have two and three UIMs, respectively [5]. The additional UIM domain is thought to be the binding site for UBL domain-containing proteins. The UBL domain in RAD23A preferentially binds to UIM-2 in Rpn10 [6], whereas the UBL domain in UBQLN2 exhibits a 25-fold stronger affinity for the N-terminal UIM-1 over UIM-2 in hRpn10 [7]. In plants, UIM-2 specifically interacts with ATG8 for the autophagic turnover of the proteasome, or proteaphagy [8]. Interestingly, recent studies have suggested that the UBL domains in both RAD23 and UBQLN can activate the mammalian proteasome even though they bind to different UIMs in Rpn10 [9,10]. It remains unclear how these UIM domains are coordinated as a platform for ubiquitin chains and UBL domain-containing proteins. Mice lacking all the UIMs in Rpn10 exhibit embryonic lethality, suggesting the importance of UIMs in Rpn10 [11].

Rpn13/ADRM1 binds ubiquitin via a conserved antiparallel $\beta$-sheet region, termed the Pru domain (Pleckstrin-like receptor for ubiquitin), which itself binds to Lys48-linked diubiquitin with an affinity of $\sim 90 \mathrm{nM}$ [12]. Rpn13 Pru also binds to UBL domain-containing proteins. NMR analysis revealed that Rpn13 directly binds to RAD23A and UBQLN2, and the Kd value of Rpn13 Pru-RAD23A UBL domain is about $36 \mu \mathrm{M}$ [12]. Rpn13 also binds to the Uch37/UCHL5 deubiquitylating enzyme via its carboxy-terminal domain, called the deubiquitinase adapter (DEUBAD) domain. Curiously, Rpn13 in budding yeast lacks both the DEUBAD domain and Uch37 [13]. By trimming the ubiquitin chains, Uch37 may remodel ubiquitin chains on ubiquitin receptors.

Rpn1 is a large proteasome subunit composed of 11 repeats of 30 to 40 residues, known as proteasome/cyclosome (PC) repeats, each of which forms a helix-turn-helix hairpin [14,15]. These PC repeats form a closed toroid domain consisting of T1 and T2 sites. T1 binds to both ubiquitin and the RAD23 UBL domain, and T2 recognizes the UBL domain of deubiquitylating enzyme Ubp6/USP14 [16]. Thus, the toroid domain in Rpn1 serves as the receptor not only for ubiquitylated proteins but also for shuttling factors and deubiquitylating enzymes.

It has been suggested that, to a certain extent, these ubiquitin receptors have functional redundancy in the ubiquitin recognition of the proteasome. Single gene mutation of the ubiquitin- and UBL domain-binding domains of these ubiquitin receptors is permissive in yeast. In addition, yeast cells that lack all three ubiquitin-binding sites are still viable, although they exhibit amino-acid analog sensitivity [16]. Using a well-defined model substrate and mutant proteasomes, Martinez-Fonts et al. elegantly demonstrated that Rpn10 is a primary receptor for Lys48-linked ubiquitylated substrates, and Rpn1 and Rpn13 are co-receptors for multi-ubiquitylated substrates [17]. Moreover, a recent study showed that Rpn1 binds Lys11/Lys48 branched ubiquitin chains more efficiently than Lys48-linked homotypic chains [18]. Thus, three receptors provide a versatile platform for various ubiquitin chain topologies. In mice, the liver-specific deletion of either RPN10 or RPN13 exhibited modest tissue impairment, but the simultaneous loss of both RPN10 and RPN13 caused severe liver injury accompanied by a massive accumulation of ubiquitin conjugates and UBL domain-containing proteins on the proteasome [19].

Other subunits, such as Rpt5 and Rpn15/Sem1/Dss1, were proposed to bind to ubiquitin [20,21]. However, this process is not yet fully understood.

\subsection{UBL-UBA Proteins}

Some ubiquitylated proteins are recognized and degraded by the proteasome with the help of UBL-UBA domain-containing proteins (UBL-UBA proteins). The main members of the UBL-UBA family are Rad23, also known as RAD23A/B in mammals; Dsk2, also known as UBQLN1 4 and L in mammals; and Ddi1/2. The UBL-UBA proteins contain a UBL domain in the N-terminus and one or two UBA domains in the C-terminus. These proteins simultaneously bind to ubiquitin via UBA 
domains $[22,23]$ and to the proteasome via the UBL domain, and they shuttle ubiquitylated substrates to the proteasome. Like ubiquitin chains, the UBL domain binds to Rpn1, Rpn10, or Rpn13.

Rad23, which is conserved from yeast to humans, contains an N-terminal UBL domain, a stress inducible-1 (STI) domain (Rad4/XPC binding), and two UBA domains. Rad23 was originally identified as a nucleotide excision repair factor that is involved in DNA repair [24], and it is also characterized as a regulatory factor of intracellular protein degradation. The STI domain is also known to bind $N$-glycanase (Png1 in yeast, NGLY1/2 in mammals), suggesting the involvement of the proteasomal degradation of the glycoprotein that has leaked into the cytoplasm [25]. RAD23A and RAD23B in mammals show high homology in their amino acid sequences and therefore they can functionally compensate for one another's roles in nucleotide excision repair [26]. Despite this functional redundancy of RAD23A and B, only RAD23B-deficient mice exhibited developmental impairment and growth retardation [26]. The susceptibility of each protein's depletion in mice may be explained by the difference in protein expression levels in their tissues. Indeed, the expression level of RAD23B is 6- to 10-fold higher than that of RAD23A in cultured human cells, suggesting that the expression levels of these proteins are differentially controlled depending on cell or tissue type.

Dsk2 was originally identified, together with Rad23, as an essential factor for the duplication of the microtubule organization center [27]. Dsk2 contains a UBL domain, an STI domain that binds to heat shock proteins, and a UBA domain. Five UBQLN proteins (UBQLN1 4 and L) are encoded in the human genome. UBQLN1, 2, and 4 have some redundant functions [28], whereas UBQLN3 is mainly expressed in the testis, and UBQLN-L lacks the UBA domain. The STI domain of UBQLNs appears to directly recognize transmembrane proteins, and with their associated ubiquitin E3 ligases, UBQLNs target mislocalized transmembrane proteins that fail to be transported to the endoplasmic reticulum (ER) or mitochondria for proteasomal degradation [29,30]. In addition, the STI domain interacts with the disaggregase HSP70-HSP110 and shuttles aggregated proteins to the proteasome [31]. Notably, mutations in the UBQLN2 gene are linked to amyotrophic lateral sclerosis (ALS) and frontotemporal lobar degeneration [32]. UBQLN4 also participates in the export of ubiquitylated proteins from the nucleus to the cytoplasm through association with an nuclear export signal-containing protein, named POST, and may contribute to nuclear protein quality control [33]. Yeast Rad23 and Dsk2 play a redundant role in proteasomal degradation $[34,35]$ and synergistically contribute to shuttling ubiquitylated substrates to the proteasome [4]. However, human RAD23A/B and UBQLN proteins seem to have some specific functions, e.g., RAD23B and UBQLN proteins contribute to droplet formation by the proteasome [36] (described below) and autophagosome [37], respectively.

Ddi1 (also known as DDI1/2 in mammals) remains less understood. Ddi1 has a helical domain of Ddi1 and a predicted retroviral protease-like domain that has a similar amino acid sequence to retroviral aspartyl proteases; for example, those encoded in HIV [38,39]. Interestingly, the UBL domain of Ddi1 can bind not only to the proteasome but also to ubiquitin, as can the UBA domain [40,41]. Interestingly, recent studies have revealed that yeast Ddi1 is a ubiquitin-dependent protease that preferentially recognizes long Lys48-linked ubiquitin chains and cleaves proteasome substrates [42]. In mammals, the cleavage of DDI2 activates an ER-resident Nrf1 transcription factor that regulates the gene expression of proteasome subunits to compensate for proteasome dysfunction [43].

\subsection{Cdc48/p97/VCP-Ufd1-Npl4 Complex}

Cdc48 (also known as p97 or VCP in mammals) is a hexameric AAA-type ATPase that is essential for cell survival. p97 functions in many cellular processes, such as ER-associated degradation (ERAD), selective autophagy, and DNA damage responses [44,45]. Multiple different cofactors regulate p97 and generate functional diversity [44]. The Ufd1 (UFD1L in humans)-Np14 (NPLOC4 in humans) heterodimer is the best-characterized ubiquitin-binding cofactor in proteasomal degradation. Polyubiquitylated proteins which are embedded in membranes or assembled in multi-subunit complexes are extracted by the p97-Ufd1-Np14 complex using ATP hydrolysis. Human Npl4 consists of an N-terminal ubiquitin-X (UBX)-like domain that binds to p97, a zinc-finger domain (ZF-Np14), an Mpr1/Pad1 N-terminal 
(MPN) domain found in JAB1/MPN/Mov34 metalloenzyme (JAMM)-family deubiquitylating enzymes, a C-terminal domain (CTD), and an Npl4 zinc finger (NZF) domain (Figure 2). In mammals, Npl4 binds to ubiquitin via the NZF domain without ubiquitin chain selectivity [46-48]. Interestingly, yeast Npl4 lacks the NZF domain, and instead, the ZF-Np14-MPN-CTD is utilized for ubiquitin binding [4,47]. Since the MPN domain in Npl4 is topologically similar to the catalytic domain of JAMM-family deubiquitylating enzymes, the MPN domain was thought to be the binding site for ubiquitin. However, recent cryo-electron microscopy analysis has shown that the Lys48 ubiquitin chain does not interact with the groove of the MPN domain in yeast Npl4 but rather with the CTD domain and other regions of the MPN domain [49]. The crystal structures of yeast Npl4 in the complex with Lys48-linked diubiquitin revealed that the C-terminal helix and N-terminal loop of CTD mainly interact with the distal and proximal ubiquitin moieties, respectively [50]. Ufd1, rather than ubiquitin, occupies a hydrophobic groove of the MPN domain of Npl4 [50]. Ufd1 also has two short small heterodimer partner (SHP) motifs that bind to p97, and it contains the Ufd1 truncation 3 (UT3) domain that binds to ubiquitin.

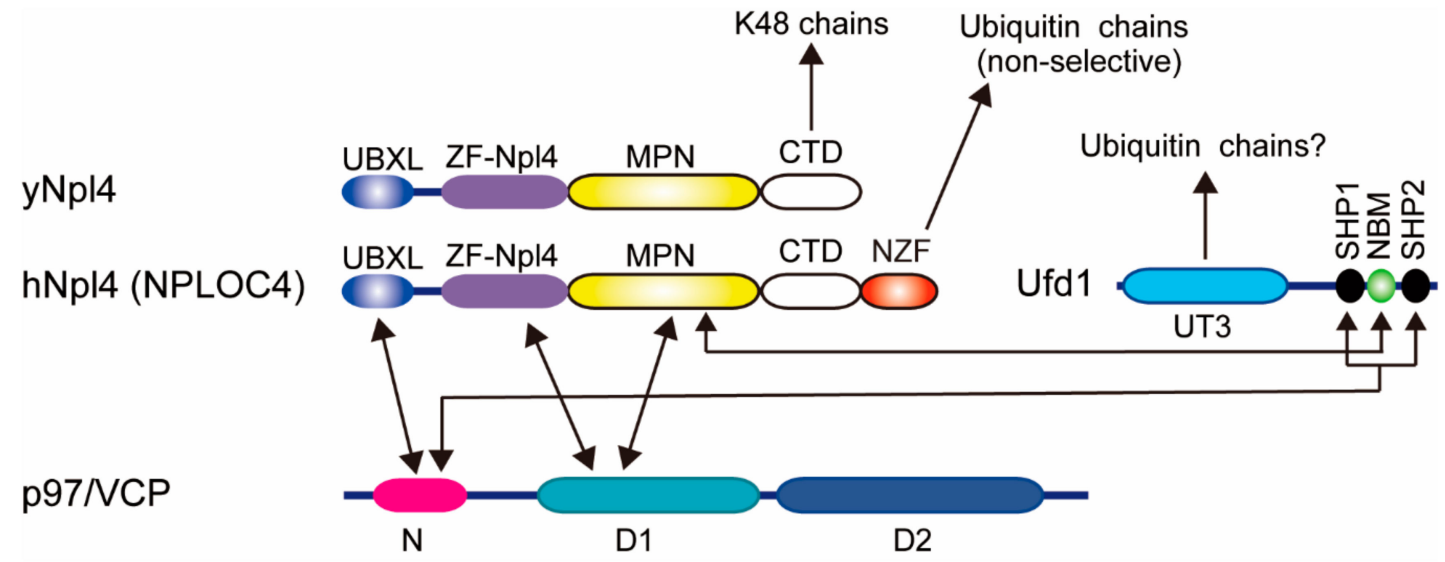

Figure 2. Domain architectures of p97, Ufd1, and Np14. Known interactions are indicated by arrows. UBXL, ubiquitin regulatory X (UBX)-like. ZF, Zinc finger. MPN, Mpr1/Pad1 N-terminal. CTD, C-terminal domain. NZF, Npl4 zinc finger. NBM, Npl4-binding motif. SHP, small heterodimer partner.

\subsection{The Major Pathway for Proteasomal Degradation}

Since the proteasome itself contains the ubiquitin receptors Rpn1, Rpn10, and Rpn13, it was thought that the proteasome directly recognizes ubiquitylated substrates. Nevertheless, the degradation of some ubiquitylated substrates requires the UBL-UBA proteins and p97 prior to recognition by the proteasome $[34,35,51,52]$. The importance of the UBL-UBA proteins and p97 in proteasomal degradation has been a long-standing question. Quantitative proteomics revealed that proteasome-bound ubiquitin chains were dramatically decreased in rad23 and dsk2 knockout cells [4], suggesting that most proteasome substrates are degraded through the Rad23- and Dsk2-dependent pathway. In a p97 temperature-sensitive mutant ( $c d c 48-3)$, ubiquitylated substrates showed significant accumulation on the proteasome. Surprisingly, this accumulation was almost completely abolished by the knockout of rad23 and dsk2, suggesting that Rad23 and Dsk2 are downstream factors of p97, reminiscent of the escort model originally described by Richly et al. [34]. These findings indicated that the p97-Rad23/Dsk2 axis plays a major role in ubiquitin-dependent proteasomal degradation rather than in direct proteasomal recognition and degradation. Recently, two independent groups reported that the p97-Ufd1-Np14 complex has unfoldase activity that relies on ATP hydrolysis and substrate ubiquitylation $[53,54]$. Moreover, efficient proteasomal degradation requires the presence of an unstructured region in substrates, which facilitates proteasomal engagement with the substrates and initiates unfolding and degradation [55]. Tomita et al. reported that calpain cleavage exposes a region that is recognized by the proteasome and allows it to initiate degradation efficiently [56]. In this 
context, the proteasome has difficulty degrading well-folded proteins that lack an unstructured region; therefore, the proteasome needs the assistance of the p97-Ufd1-Npl4 complex [57]. It is assumed that p97 provides the initiation site for the proteasome, and thus the proteasome can efficiently degrade a large number of proteins in cells.

\section{Ubiquitin Signal for the Proteasome: Linkage Type and Length}

\subsection{Ubiquitin Chain Type Selectivity for Proteasomal Degradation}

It is widely known that the tetra-Lys48-linked chain is the primary signal in substrate degradation by the proteasome [58]. Curiously, neither the proteasome itself nor Rad23, Dsk2, or Ddi1 show strict selectivity for Lys48 chains in vitro [59,60]. Recent studies have suggested that various ubiquitin architectures can also target proteins for proteasomal degradation. Multiple mono-ubiquitylation can efficiently induce proteasomal degradation, at least in vitro [61]. Additionally, multiple short chains with Lys11, Lys48, and Lys63 linkages can efficiently induce proteasomal degradation [61,62]. Moreover, in mammalian cells, anaphase-promoting complex/cyclosome (APC/C) substrates modified with Lys11/Lys48 branched chains are degraded more efficiently than those with homogenous Lys48-linked chains [63]. In yeast, Lys29/Lys48 branched chains attached to ubiquitin fusion degradation substrates accelerate proteasomal degradation [64]. Lys63/Lys48 branched chains also trigger proteasomal degradation [65]. To clarify what type of ubiquitin signal is primarily recognized by the proteasome in vivo, we performed a quantitative proteomic analysis of the ubiquitin linkage-type selectivity of major ubiquitin-binding domain (UBD)-containing proteins and the proteasome in yeast [4]. Mass spectrometry-based quantification revealed that the proteasome mainly binds Lys29 and Lys48 chains (about 7\% and 90\%, respectively), suggesting that these chains are the primary signals in yeast cells. A comparative analysis of the ubiquitin linkage selectivity of 14 major UBD proteins in yeast revealed that the UBL-UBA proteins and all p97 cofactors showed strong preferences for Lys48 linkage, whereas UBD proteins related to endocytosis were associated with K63 linkage. We also found that Np14 had strict selectivity for the Lys48-linked chains. Given that all upstream factors for the proteasome were highly selective for Lys48- and Lys29-linked chains in vivo, but only Np14 had Lys48 chain selectivity in vitro, we propose that the p97-Ufd1-Np14 complex rather than Rad23 and Dsk2 determines the Lys48-linkage selectivity for proteasomal degradation in cells. Notably, although the abundance of Lys11 linkage was estimated to be very low (comprising only $1.3 \%$ of the eight different linkages) in our experimental setting, a more recent study demonstrated that Lys11/Lys48-mixed ubiquitin chains are utilized for the proteasomal degradation of misfolded proteins in the cytoplasm [66]. In mammalian cells, mass spectrometry-based quantification revealed that the human proteasome contains relatively high levels of Lys11 and Lys63 linkages, probably derived from Lys11/Lys48 and Lys48/Lys63 branched chains [65], clearly suggesting that proteasome-targeting signals are more complex in higher eukaryotes.

\subsection{Ubiquitin Chain Length as a Signal for Proteasomal Degradation}

Ubiquitin chain length is also an important factor for ubiquitin signaling. Historically, it has been thought that the Lys48 chain is the primary signal for proteasomal degradation. An in vitro analysis revealed that four or more ubiquitin molecules on substrate proteins are sufficient to target proteins to the proteasome [58]. However, a recent study suggested that various chain lengths contribute to substrate degradation in vitro. A single-molecule kinetic analysis showed that the proteasome degrades cyclin B2 with multiple short chains [61,62]. The authors visualized the activity of the APC, a ubiquitin ligase that plays an important role in the cell cycle. The APC/C rapidly attached monoto tri-ubiquitin molecules to cyclin B2 [61,62]. In addition, mono-ubiquitylated small disordered proteins (<150 amino acids) are sufficiently degraded by the proteasome [67]. Nuclear magnetic resonance studies demonstrated that Rpn10 and Rpn13 simultaneously bind to ubiquitin chains, with a preference for Rpn10 for distal ubiquitin and Rpn13 for proximal ubiquitin [68]. Cryo-electron 
microscopy analysis revealed that the distance between Rpn10 and Rpn13 is approximately $100 \AA$, which is the same length as a tetra-ubiquitin chain [69]. One possible scenario is that this distance determines the minimum length for a ubiquitin chain recognized by the proteasome. Despite the fundamental importance of each substrate's ubiquitin chain length, there have been no methods to determine it, other than through estimation via gel mobility on sodium dodecyl sulfate polyacrylamide gel electrophoresis (SDS-PAGE). To determine the length of ubiquitin chains in biological samples, we designed an approach using trypsin and a ubiquitin chain protector, named "ubiquitin chain protection from trypsinization (Ub-ProT)" [70]. In native conditions, trypsin preferentially cleaves at Arg74 of ubiquitin [71], but substrate-attached chains are protected from trypsinization in the presence of a ubiquitin chain protector such as trypsin resistant tandem ubiquitin-binding entity (TR-TUBE) [70,72]. The Ub-ProT method, using soluble fractions obtained from yeast, revealed that the substrate-attached ubiquitins mainly ranged from monomers to heptamers [70]. Interestingly, the maximal ubiquitin chain length hardly changed as a result of proteasome inhibition, suggesting that the mechanism for regulating the ubiquitin chain length lies upstream of the proteasome in cells. By contrast, in p97 and Np14 mutants, the global ubiquitin chain length was elongated, suggesting that the recognition and segregation of ubiquitylated substrates by p97-Ufd1-Np14 are the key steps for determining ubiquitin chain length in cells.

On the basis of these findings, we proposed a model for ubiquitin code in proteasomal degradation (Figure 3). In this model, the p97-Ufd1-Np14 complex is the factor that is most upstream of the proteasome and is involved in recognizing Lys48-linked ubiquitin conjugates; in particular, Np14 governs the Lys48 selectivity of proteasomal degradation. p97-dependent segregation may antagonize ubiquitylation and terminate ubiquitin chain elongation on the substrate complex. It can be hypothesized that Np14 recognizes and segregates Lys48 chains (which are roughly heptamers in length) on the substrate. Rad23 and Dsk2 then capture the substrates and deliver them to the proteasome. Twomey et al. demonstrated that otu1, a p97-associated deubiquitylating enzyme, cleaves ubiquitin chains on the substrate and requires efficient substrate unfolding by p97. It remains unclear how deubiquitylated substrates are handed over to shuttle factors. Besides, the decrease in the segregation activity of mutant p97 results in the elongation of the ubiquitin chain on the substrates. It appears that substrates that remain in complexes can be transported to the proteasome and inhibit proteasome activity by competing with authentic ubiquitylated substrates.

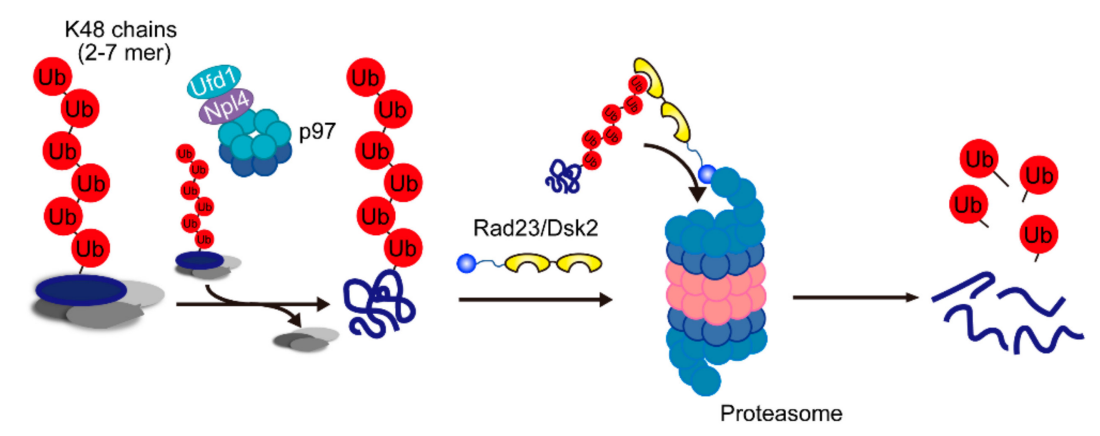

Figure 3. A model of the major route for degradation of proteasomal substrates. Two- to seven-mer Lys48 chains attached on the substrate are recognized by Npl4, and the substrate is segregated by p97. Then, the segregated substrate is transferred to shuttle factors and delivered to the proteasome.

\section{Intracellular Dynamics of the Proteasome}

\subsection{Discovery of Stress-Dependent Proteasome Nuclear Foci}

The proteasome is localized in both the cytoplasm and the nucleus. In particular, in proliferating yeast and mammalian cells, the proteasome is highly enriched in the nucleus and is presumably involved in the degradation of nuclear proteins [73-75]. When nuclear proteasomes are eliminated in yeast by a 
chemically-induced dimerization system, ubiquitylated proteins show significant accumulation [76]. However, the biological importance of proteasomal degradation in the nucleus under basal conditions is not well characterized. Abnormal proteins, such as those that are misfolded, are presumably constantly produced and then degraded by the proteasome, not only in the cytoplasm but also in the nucleus. During our microscopic analysis of the proteasome, we unexpectedly observed that proteasomes rapidly formed multiple foci in the nucleus upon hyperosmotic stress [36]. These proteasome foci were transient structures that emerged within a minute after hyperosmotic stimulation and disappeared after a few hours. Cryo-electron tomographic analysis successfully captured the clustering of proteasomes in the nucleus upon osmotic stimulation. We investigated whether these proteasome foci consisted of droplets, as they contained no aggregates or scaffold proteins. Indeed, these foci had droplet-like properties: they consisted of small foci that fused into larger foci, their shape was almost spherical, and they dissolved following the addition of 1,6-hexanediol, an aliphatic alcohol that destabilizes liquid droplets. Proteasome foci did not co-localize with known nuclear structures, such as promyelocytic leukemia bodies or Cajal bodies, suggesting that the nuclear proteasome foci induced by hyperosmotic stress have distinct roles. Liquid-liquid phase separation (LLPS) has become a hot topic in the life sciences in recent years. Cellular structures formed through LLPS are called membrane-less organelles or biomolecular condensates. These include stress granules, cytosolic p-bodies, and nuclear speckles [77]. LLPS is a rapid, reversible, and widespread compartmentalization mechanism in cells. Distinct subcompartments facilitate the spatiotemporal regulation of biological reactions [77]. We found that proteasome foci mainly co-localized with Lys48-linked ubiquitin chains and did not form in cells that were treated with the E1 inhibitor. By contrast, the number and size of foci increased and their clearance was delayed in cells treated with a proteasome inhibitor or p97 inhibitor. Thus, ubiquitylated substrates are required for the formation of proteasome foci and proteasomal degradation is necessary for their clearance, suggesting that proteasome foci are the proteolytic sites of ubiquitylated proteins. Proteomics analysis revealed that the ubiquitylation level of several housekeeping proteins, including histones, HSP90 (HSP90AB1 and HSP90AA1), and ribosomal proteins, increased under hyperosmotic stress, suggesting that this stress has global effects on the cellular proteome. In cultured cells, about 75,000 ribosome subunit molecules are synthesized per minute, and orphan subunits that fail to be incorporated into ribosomes are degraded by the proteasome [78-80]. Electron microscopic analysis showed that the nucleolar dense fibrillar compartment, where pre-ribosomes are assembled, was disrupted under hyperosmotic stress. Hyperosmotic stress also inhibited ribosome assembly in the nucleus, suggesting that the assembly process is susceptible to this condition. Some ribosomal proteins (RPL15, RPL29) aggregated in the nucleoplasm upon hyperosmotic stress and merged with proteasome foci, and they subsequently disappeared within an hour. These findings suggest that orphan ribosomal subunits are one of the major types of substrates in proteasome foci under hyperosmotic stress.

\subsection{Molecular Mechanism of the Formation of Proteasome Foci}

We also revealed that some proteasome-interacting proteins, including p97, RAD23B, and the ubiquitin ligase UBE3A (also known as E6-AP), co-localized to proteasome foci. The knockout of UBE3A reduced the number of proteasome foci formed upon hyperosmotic stress, suggesting that UBE3A regulates the proteasome itself or the ubiquitylation of substrate proteins in the foci [81], but the underlying molecular mechanism remains unknown. On the other hand, the proteasome foci were not detected in RAD23B knockout cells. We first assumed that the ubiquitylated proteins form foci without RAD23B. However, neither proteasomes nor ubiquitin formed foci in RAD23B knockout or mutated in the UBA domain cells, suggesting that the ubiquitin-binding ability of RAD23B induces the phase separation of ubiquitylated proteins. Proteins that induce LLPS can be classified into two groups. One consists of denatured proteins with intrinsically disordered regions (IDRs) such as fused in sarcoma (FUS) or tar DNA binding protein-43 (TDP-43); these proteins phase-separate with RNA $[82,83]$. Others cause phase separation due to the multivalent interaction of molecules which use multiple domains like the Src homology 3 (SH3) domain and the proline-rich motif [84]. 
RAD23B has two UBA domains, so it can bind to two ubiquitin chains. Each UBA domain weakly interacts with a ubiquitin monomer, specifically in a 1:1 molecular ratio, but it is known that the avidity increases exponentially when ubiquitin forms a chain [85]. We observed the co-phase separation of RAD23B and Lys48 chains in vitro. RAD23B prefers ubiquitin chains with four or more ubiquitin molecules, and both UBA domains of RAD23B are required for droplet formation. Therefore, the LLPS of proteasomes, ubiquitin chains, and RAD23B is mediated through multivalent interactions between polyubiquitin and RAD23B.

Based on these findings, we proposed a model for the formation of proteasome foci (Figure 4). Hyperosmotic stress increases molecular crowding and accumulates ubiquitylated proteins such as ribosomal proteins. Consequently, RAD23B and ubiquitylated substrates form liquid droplets by LLPS, and proteasomes and their cofactors are recruited to the foci. We assume that the proteasome foci are the sites for the sequestration and efficient degradation of ubiquitylated proteins in the nucleus. Like RAD23B, UBQLN is also linked to LLPS, though it is a completely different mechanism. UBQLN2 is recruited to stress granules and undergoes LLPS without ubiquitin [86]. The LLPS is promoted by multivalent interactions across the IDR and the UBA domain of UBQLN2.

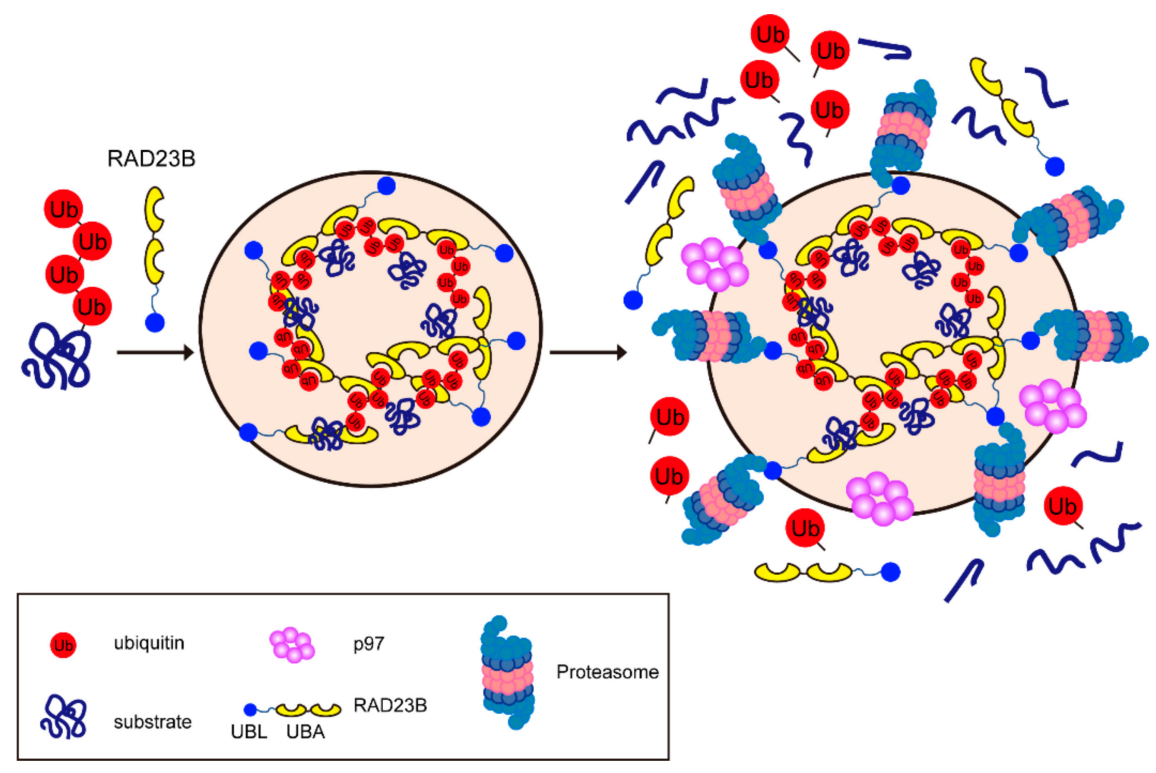

Figure 4. Hyperosmotic stress-induced proteasome foci in the nucleus. The multivalent interactions between polyubiquitin chains and the UBA of RAD23B drive the liquid-liquid phase separation (LLPS). Then, the proteasomes and $\mathrm{p} 97$ are recruited to the foci and degrade the ubiquitylated substrates.

Interestingly, ubiquitin binding disrupts the UBQLN2 LLPS, suggesting that ubiquitin and UBQLN2 interactions result in the concomitant extraction of the polyubiquitylated substrate from stress granules. Importantly, some ALS-linked UBQLN mutations promote UBQLN2 oligomerization and accelerate LLPS formation in vitro [87], implying that the solidification of UBQLN2 directly links to ALS' pathology. It has been reported that autophagy, another proteolytic system, also utilizes ubiquitin chain-dependent phase separation. The ubiquitin-selective autophagy adapter p62 has a Phox and Bem1 (PB1) domain, a UBA domain, and an LC3-binding domain for oligomer formation. p62 collects ubiquitylated substrates upon proteasome inhibition and oxidative stress, forms a droplet structure, and facilitates autophagy. The formation of these droplets (called p62 bodies) requires polyubiquitin chains linked with eight or more ubiquitins, and the UBA domain in p62 is important for the formation of p62 bodies [88]. Thus, one of the biological roles of polyubiquitin chain formation may be to induce LLPS. Since more than 100 proteins with UBDs are encoded in the human genome and many have multiple UBDs, a distinct LLPS mechanism is likely used by each ubiquitin-dependent pathway. 


\section{Conclusions and Perspective}

Emerging evidence, reviewed in this article, emphasizes the crucial steps of substrate extraction/segregation and transport that are mediated by p97 and shuttling factors for proteasomal degradation. However, further studies are needed to elucidate how these steps together impact the ubiquitylated substrate to achieve effective degradation. In addition, the spatiotemporal and elaborate regulation of proteasomes is important for cell responses to the intra- and extracellular environment, since the proteasome plays a fundamental role in protein homeostasis. Since a number of proteasome-containing structures are known [89-92], the discovering of the osmotic stress-inducing proteasome phase separation sheds light on the importance of proteasome dynamics in response to the cellular environment. As the intracellular density of biomolecules is very high, the compartmentation of proteasomes and substrates with the help of shuttling factors may facilitate efficient proteasomal degradation. We expect that research over the next decade on the molecular mechanisms of substrate delivery and proteasome dynamics will contribute to a comprehensive understanding of the ubiquitinproteasome system.

Author Contributions: H.T. and Y.S. wrote the original draft; A.E. and Y.S. edited the manuscript. All authors have read and agreed to the published version of the manuscript.

Funding: This research was supported by AMED under Grant Numbers JP18gm1110003 (Y.S) and by MEXT/JSPS KAKENHI Grant Numbers JP18K19352 (Y.S.), JP20H03214 (H.T.), JP18K14913 (H.T.), JP26293018 (Y.S.), and JP18H05498 (Y.S.).

Conflicts of Interest: The authors declare no conflict of interest.

\section{References}

1. Collins, G.A.; Goldberg, A.L. The Logic of the 26S Proteasome. Cell 2017, 169, 792-806. [CrossRef] [PubMed]

2. Kish-Trier, E.; Hill, C.P. Structural biology of the proteasome. Annu. Rev. Biophys. 2013, 42, 29-49. [CrossRef] [PubMed]

3. Glickman, M.H.; Ciechanover, A. The ubiquitin-proteasome proteolytic pathway: Destruction for the sake of construction. Physiol. Rev. 2002, 82, 373-428. [CrossRef] [PubMed]

4. Tsuchiya, H.; Ohtake, F.; Arai, N.; Kaiho, A.; Yasuda, S.; Tanaka, K.; Saeki, Y. In Vivo Ubiquitin Linkage-type Analysis Reveals that the Cdc48-Rad23/Dsk2 Axis Contributes to K48-Linked Chain Specificity of the Proteasome. Mol. Cell 2017, 66, 488-502. [CrossRef]

5. Fatimababy, A.S.; Lin, Y.L.; Usharani, R.; Radjacommare, R.; Wang, H.T.; Tsai, H.L.; Lee, Y.; Fu, H. Cross-species divergence of the major recognition pathways of ubiquitylated substrates for ubiquitin/26S proteasome-mediated proteolysis. FEBS J. 2010, 277, 796-816. [CrossRef]

6. Kang, Y.; Chen, X.; Lary, J.W.; Cole, J.L.; Walters, K.J. Defining how ubiquitin receptors hHR23a and S5a bind polyubiquitin. J. Mol. Biol. 2007, 369, 168-176. [CrossRef]

7. Chen, X.; Ebelle, D.L.; Wright, B.J.; Sridharan, V.; Hooper, E.; Walters, K.J. Structure of hRpn10 Bound to UBQLN2 UBL Illustrates Basis for Complementarity between Shuttle Factors and Substrates at the Proteasome. J. Mol. Biol. 2019, 431, 939-955. [CrossRef]

8. Marshall, R.S.; Li, F.; Gemperline, D.C.; Book, A.J.; Vierstra, R.D. Autophagic Degradation of the 26S Proteasome Is Mediated by the Dual ATG8/Ubiquitin Receptor RPN10 in Arabidopsis. Mol. Cell 2015, 58, 1053-1066. [CrossRef]

9. Kim, H.T.; Goldberg, A.L. UBL domain of Usp14 and other proteins stimulates proteasome activities and protein degradation in cells. Proc. Natl. Acad. Sci. USA 2018, 115, E11642-E11650. [CrossRef]

10. Collins, G.A.; Goldberg, A.L. Proteins containing ubiquitin-like (Ubl) domains not only bind to $26 \mathrm{~S}$ proteasomes but also induce their activation. Proc. Natl. Acad. Sci. USA 2020, 117, 4664-4674. [CrossRef]

11. Hamazaki, J.; Sasaki, K.; Kawahara, H.; Hisanaga, S.; Tanaka, K.; Murata, S. Rpn10-mediated degradation of ubiquitinated proteins is essential for mouse development. Mol. Cell Biol. 2007, 27, 6629-6638. [CrossRef] [PubMed] 
12. Husnjak, K.; Elsasser, S.; Zhang, N.; Chen, X.; Randles, L.; Shi, Y.; Hofmann, K.; Walters, K.J.; Finley, D.; Dikic, I. Proteasome subunit Rpn13 is a novel ubiquitin receptor. Nature 2008, 453, 481-488. [CrossRef] [PubMed]

13. Yao, T.; Song, L.; Xu, W.; DeMartino, G.N.; Florens, L.; Swanson, S.K.; Washburn, M.P.; Conaway, R.C.; Conaway, J.W.; Cohen, R.E. Proteasome recruitment and activation of the Uch37 deubiquitinating enzyme by Adrm1. Nat. Cell Biol. 2006, 8, 994-1002. [CrossRef]

14. Kajava, A.V. What curves alpha-solenoids? Evidence for an alpha-helical toroid structure of Rpn1 and Rpn2 proteins of the $26 \mathrm{~S}$ proteasome. J. Biol. Chem. 2002, 277, 49791-49798. [CrossRef]

15. He, J.; Kulkarni, K.; da Fonseca, P.C.; Krutauz, D.; Glickman, M.H.; Barford, D.; Morris, E.P. The structure of the 26S proteasome subunit Rpn2 reveals its PC repeat domain as a closed toroid of two concentric alpha-helical rings. Structure 2012, 20, 513-521. [CrossRef]

16. Shi, Y.; Chen, X.; Elsasser, S.; Stocks, B.B.; Tian, G.; Lee, B.H.; Shi, Y.; Zhang, N.; de Poot, S.A.; Tuebing, F.; et al. Rpn1 provides adjacent receptor sites for substrate binding and deubiquitination by the proteasome. Science 2016, 351. [CrossRef] [PubMed]

17. Martinez-Fonts, K.; Davis, C.; Tomita, T.; Elsasser, S.; Nager, A.R.; Shi, Y.; Finley, D.; Matouschek, A. The proteasome $19 \mathrm{~S}$ cap and its ubiquitin receptors provide a versatile recognition platform for substrates. Nat. Commun. 2020, 11, 477. [CrossRef] [PubMed]

18. Boughton, A.J.; Krueger, S.; Fushman, D. Branching via K11 and K48 Bestows Ubiquitin Chains with a Unique Interdomain Interface and Enhanced Affinity for Proteasomal Subunit Rpn1. Structure 2020, 28, 29-43.e26. [CrossRef]

19. Hamazaki, J.; Hirayama, S.; Murata, S. Redundant Roles of Rpn10 and Rpn13 in Recognition of Ubiquitinated Proteins and Cellular Homeostasis. PLoS Genet. 2015, 11, e1005401. [CrossRef]

20. Lam, Y.A.; Lawson, T.G.; Velayutham, M.; Zweier, J.L.; Pickart, C.M. A proteasomal ATPase subunit recognizes the polyubiquitin degradation signal. Nature 2002, 416, 763-767. [CrossRef]

21. Paraskevopoulos, K.; Kriegenburg, F.; Tatham, M.H.; Rosner, H.I.; Medina, B.; Larsen, I.B.; Brandstrup, R.; Hardwick, K.G.; Hay, R.T.; Kragelund, B.B.; et al. Dss1 is a 26S proteasome ubiquitin receptor. Mol. Cell 2014, 56, 453-461. [CrossRef] [PubMed]

22. Wilkinson, C.R.; Seeger, M.; Hartmann-Petersen, R.; Stone, M.; Wallace, M.; Semple, C.; Gordon, C. Proteins containing the UBA domain are able to bind to multi-ubiquitin chains. Nat. Cell Biol. 2001, 3, 939-943. [CrossRef] [PubMed]

23. Bertolaet, B.L.; Clarke, D.J.; Wolff, M.; Watson, M.H.; Henze, M.; Divita, G.; Reed, S.I. UBA domains of DNA damage-inducible proteins interact with ubiquitin. Nat. Struct. Biol. 2001, 8, 417-422. [CrossRef] [PubMed]

24. Perozzi, G.; Prakash, S. RAD7 gene of Saccharomyces cerevisiae: Transcripts, nucleotide sequence analysis, and functional relationship between the RAD7 and RAD23 gene products. Mol. Cell Biol. 1986, 6, 1497-1507. [CrossRef] [PubMed]

25. Suzuki, T.; Park, H.; Kwofie, M.A.; Lennarz, W.J. Rad23 provides a link between the Png1 deglycosylating enzyme and the $26 \mathrm{~S}$ proteasome in yeast. J. Biol. Chem. 2001, 276, 21601-21607. [CrossRef]

26. Ng, J.M.; Vrieling, H.; Sugasawa, K.; Ooms, M.P.; Grootegoed, J.A.; Vreeburg, J.T.; Visser, P.; Beems, R.B.; Gorgels, T.G.; Hanaoka, F.; et al. Developmental defects and male sterility in mice lacking the ubiquitin-like DNA repair gene mHR23B. Mol. Cell. Biol. 2002, 22, 1233-1245. [CrossRef]

27. Biggins, S.; Ivanovska, I.; Rose, M.D. Yeast ubiquitin-like genes are involved in duplication of the microtubule organizing center. J. Cell Biol. 1996, 133, 1331-1346. [CrossRef]

28. Kang, Y.; Zhang, N.; Koepp, D.M.; Walters, K.J. Ubiquitin receptor proteins hHR23a and hPLIC2 interact. J. Mol. Biol. 2007, 365, 1093-1101. [CrossRef]

29. Itakura, E.; Zavodszky, E.; Shao, S.; Wohlever, M.L.; Keenan, R.J.; Hegde, R.S. Ubiquilins Chaperone and Triage Mitochondrial Membrane Proteins for Degradation. Mol. Cell 2016, 63, 21-33. [CrossRef]

30. Suzuki, R.; Kawahara, H. UBQLN4 recognizes mislocalized transmembrane domain proteins and targets these to proteasomal degradation. EMBO Rep. 2016, 17, 842-857. [CrossRef]

31. Hjerpe, R.; Bett, J.S.; Keuss, M.J.; Solovyova, A.; McWilliams, T.G.; Johnson, C.; Sahu, I.; Varghese, J.; Wood, N.; Wightman, M.; et al. UBQLN2 Mediates Autophagy-Independent Protein Aggregate Clearance by the Proteasome. Cell 2016, 166, 935-949. [CrossRef] [PubMed] 
32. Deng, H.-X.; Chen, W.; Hong, S.-T.; Boycott, K.M.; Gorrie, G.H.; Siddique, N.; Yang, Y.; Fecto, F.; Shi, Y.; Zhai, H.; et al. Mutations in UBQLN2 cause dominant X-linked juvenile and adult-onset ALS and ALS/dementia. Nature 2011, 477, 211-215. [CrossRef] [PubMed]

33. Hirayama, S.; Sugihara, M.; Morito, D.; Iemura, S.I.; Natsume, T.; Murata, S.; Nagata, K. Nuclear export of ubiquitinated proteins via the UBIN-POST system. Proc. Natl. Acad. Sci. USA 2018, 115, E4199-E4208. [CrossRef] [PubMed]

34. Richly, H.; Rape, M.; Braun, S.; Rumpf, S.; Hoege, C.; Jentsch, S. A series of ubiquitin binding factors connects CDC48/p97 to substrate multiubiquitylation and proteasomal targeting. Cell 2005, 120, 73-84. [CrossRef]

35. Verma, R.; Oania, R.; Graumann, J.; Deshaies, R.J. Multiubiquitin chain receptors define a layer of substrate selectivity in the ubiquitin-proteasome system. Cell 2004, 118, 99-110. [CrossRef]

36. Yasuda, S.; Tsuchiya, H.; Kaiho, A.; Guo, Q.; Ikeuchi, K.; Endo, A.; Arai, N.; Ohtake, F.; Murata, S.; Inada, T.; et al. Stress- and ubiquitylation-dependent phase separation of the proteasome. Nature 2020, 578, 296-300. [CrossRef]

37. Lee, D.Y.; Arnott, D.; Brown, E.J. Ubiquilin4 is an adaptor protein that recruits Ubiquilin1 to the autophagy machinery. EMBO Rep. 2013, 14, 373-381. [CrossRef]

38. Sirkis, R.; Gerst, J.E.; Fass, D. Ddi1, a eukaryotic protein with the retroviral protease fold. J. Mol. Biol. 2006, 364, 376-387. [CrossRef]

39. Krylov, D.M.; Koonin, E.V. A novel family of predicted retroviral-like aspartyl proteases with a possible key role in eukaryotic cell cycle control. Curr. Biol. 2001, 11, R584-R587. [CrossRef]

40. Kaplun, L.; Tzirkin, R.; Bakhrat, A.; Shabek, N.; Ivantsiv, Y.; Raveh, D. The DNA damage-inducible UbL-UbA protein Ddi1 participates in Mec1-mediated degradation of Ho endonuclease. Mol. Cell Biol. 2005, 25, 5355-5362. [CrossRef]

41. Nowicka, U.; Zhang, D.; Walker, O.; Krutauz, D.; Castañeda, C.A.; Chaturvedi, A.; Chen, T.Y.; Reis, N.; Glickman, M.H.; Fushman, D. DNA-damage-inducible 1 protein (Ddi1) contains an uncharacteristic ubiquitin-like domain that binds ubiquitin. Structure 2015, 23, 542-557. [CrossRef]

42. Yip, M.C.J.; Bodnar, N.O.; Rapoport, T.A. Ddi1 is a ubiquitin-dependent protease. Proc. Natl. Acad. Sci. USA 2020, 117, 7776-7781. [CrossRef]

43. Koizumi, S.; Irie, T.; Hirayama, S.; Sakurai, Y.; Yashiroda, H.; Naguro, I.; Ichijo, H.; Hamazaki, J.; Murata, S. The aspartyl protease DDI2 activates Nrf1 to compensate for proteasome dysfunction. eLife 2016, 5. [CrossRef] [PubMed]

44. Meyer, H.; Bug, M.; Bremer, S. Emerging functions of the VCP/p97 AAA-ATPase in the ubiquitin system. Nat. Cell Biol. 2012, 14, 117-123. [CrossRef] [PubMed]

45. Vembar, S.S.; Brodsky, J.L. One step at a time: Endoplasmic reticulum-associated degradation. Nat. Rev. Mol. Cell Biol. 2008, 9, 944-957. [CrossRef] [PubMed]

46. Kristariyanto, Y.A.; Abdul Rehman, S.A.; Campbell, D.G.; Morrice, N.A.; Johnson, C.; Toth, R.; Kulathu, Y. K29-selective ubiquitin binding domain reveals structural basis of specificity and heterotypic nature of k29 polyubiquitin. Mol. Cell 2015, 58, 83-94. [CrossRef] [PubMed]

47. Meyer, H.H.; Wang, Y.; Warren, G. Direct binding of ubiquitin conjugates by the mammalian p97 adaptor complexes, p47 and Ufd1-Np14. EMBO J. 2002, 21, 5645-5652. [CrossRef] [PubMed]

48. Sato, Y.; Yoshikawa, A.; Yamashita, M.; Yamagata, A.; Fukai, S. Structural basis for specific recognition of Lys 63-linked polyubiquitin chains by NZF domains of TAB2 and TAB3. EMBO J. 2009, 28, 3903-3909. [CrossRef]

49. Twomey, E.C.; Ji, Z.; Wales, T.E.; Bodnar, N.O.; Ficarro, S.B.; Marto, J.A.; Engen, J.R.; Rapoport, T.A. Substrate processing by the Cdc48 ATPase complex is initiated by ubiquitin unfolding. Science 2019, 365. [CrossRef]

50. Sato, Y.; Tsuchiya, H.; Yamagata, A.; Okatsu, K.; Tanaka, K.; Saeki, Y.; Fukai, S. Structural insights into ubiquitin recognition and Ufd1 interaction of Npl4. Nat. Commun. 2019, 10, 5708. [CrossRef] [PubMed]

51. Baek, G.H.; Kim, I.; Rao, H. The Cdc48 ATPase modulates the interaction between two proteolytic factors Ufd2 and Rad23. Proc. Natl. Acad. Sci. USA 2011, 108, 13558-13563. [CrossRef] [PubMed]

52. Finley, D. Recognition and processing of ubiquitin-protein conjugates by the proteasome. Annu. Rev. Biochem. 2009, 78, 477-513. [CrossRef] [PubMed]

53. Blythe, E.E.; Olson, K.C.; Chau, V.; Deshaies, R.J. Ubiquitin- and ATP-dependent unfoldase activity of P97/VCP*NPLOC4*UFD1L is enhanced by a mutation that causes multisystem proteinopathy. Proc. Natl. Acad. Sci. USA 2017, 114, E4380-E4388. [CrossRef] 
54. Bodnar, N.O.; Kim, K.H.; Ji, Z.; Wales, T.E.; Svetlov, V.; Nudler, E.; Engen, J.R.; Walz, T.; Rapoport, T.A. Structure of the Cdc48 ATPase with its ubiquitin-binding cofactor Ufd1-Npl4. Nat. Struct. Mol. Biol. 2018, 25, 616-622. [CrossRef] [PubMed]

55. Prakash, S.; Tian, L.; Ratliff, K.S.; Lehotzky, R.E.; Matouschek, A. An unstructured initiation site is required for efficient proteasome-mediated degradation. Nat. Struct. Mol. Biol. 2004, 11, 830-837. [CrossRef]

56. Tomita, T.; Huibregtse, J.M.; Matouschek, A. A masked initiation region in retinoblastoma protein regulates its proteasomal degradation. Nat. Commun. 2020, 11, 2019. [CrossRef] [PubMed]

57. Olszewski, M.M.; Williams, C.; Dong, K.C.; Martin, A. The Cdc48 unfoldase prepares well-folded protein substrates for degradation by the $26 \mathrm{~S}$ proteasome. Commun. Biol. 2019, 2, 29. [CrossRef]

58. Thrower, J.S.; Hoffman, L.; Rechsteiner, M.; Pickart, C.M. Recognition of the polyubiquitin proteolytic signal. EMBO J. 2000, 19, 94-102. [CrossRef] [PubMed]

59. Raasi, S.; Varadan, R.; Fushman, D.; Pickart, C.M. Diverse polyubiquitin interaction properties of ubiquitin-associated domains. Nat. Struct. Mol. Biol. 2005, 12, 708-714. [CrossRef] [PubMed]

60. Nathan, J.A.; Kim, H.T.; Ting, L.; Gygi, S.P.; Goldberg, A.L. Why do cellular proteins linked to K63-polyubiquitin chains not associate with proteasomes? EMBO J. 2013, 32, 552-565. [CrossRef] [PubMed]

61. Lu, Y.; Wang, W.; Kirschner, M.W. Specificity of the anaphase-promoting complex: A single-molecule study. Science 2015, 348, 1248737. [CrossRef] [PubMed]

62. Lu, Y.; Lee, B.H.; King, R.W.; Finley, D.; Kirschner, M.W. Substrate degradation by the proteasome: A single-molecule kinetic analysis. Science 2015, 348, 1250834. [CrossRef] [PubMed]

63. Meyer, H.J.; Rape, M. Enhanced protein degradation by branched ubiquitin chains. Cell 2014, 157, $910-921$. [CrossRef]

64. Liu, C.; Liu, W.; Ye, Y.; Li, W. Ufd2p synthesizes branched ubiquitin chains to promote the degradation of substrates modified with atypical chains. Nat. Commun. 2017, 8, 14274. [CrossRef] [PubMed]

65. Ohtake, F.; Tsuchiya, H.; Saeki, Y.; Tanaka, K. K63 ubiquitylation triggers proteasomal degradation by seeding branched ubiquitin chains. Proc. Natl. Acad. Sci. USA 2018, 115, E1401-E1408. [CrossRef]

66. Samant, R.S.; Livingston, C.M.; Sontag, E.M.; Frydman, J. Distinct proteostasis circuits cooperate in nuclear and cytoplasmic protein quality control. Nature 2018, 563, 407-411. [CrossRef] [PubMed]

67. Shabek, N.; Herman-Bachinsky, Y.; Buchsbaum, S.; Lewinson, O.; Haj-Yahya, M.; Hejjaoui, M.; Lashuel, H.A.; Sommer, T.; Brik, A.; Ciechanover, A. The size of the proteasomal substrate determines whether its degradation will be mediated by mono- or polyubiquitylation. Mol. Cell 2012, 48, 87-97. [CrossRef]

68. Zhang, F.; Hu, M.; Tian, G.; Zhang, P.; Finley, D.; Jeffrey, P.D.; Shi, Y. Structural insights into the regulatory particle of the proteasome from Methanocaldococcus jannaschii. Mol. Cell 2009, 34, 473-484. [CrossRef]

69. Sakata, E.; Bohn, S.; Mihalache, O.; Kiss, P.; Beck, F.; Nagy, I.; Nickell, S.; Tanaka, K.; Saeki, Y.; Förster, F.; et al. Localization of the proteasomal ubiquitin receptors Rpn10 and Rpn13 by electron cryomicroscopy. Proc. Natl. Acad. Sci. USA 2012, 109, 1479-1484. [CrossRef]

70. Tsuchiya, H.; Burana, D.; Ohtake, F.; Arai, N.; Kaiho, A.; Komada, M.; Tanaka, K.; Saeki, Y. Ub-ProT reveals global length and composition of protein ubiquitylation in cells. Nat. Commun. 2018, 9, 524. [CrossRef]

71. $\mathrm{Xu}, \mathrm{P}$;; Peng, J. Characterization of polyubiquitin chain structure by middle-down mass spectrometry. Anal. Chem. 2008, 80, 3438-3444. [CrossRef] [PubMed]

72. Ohtake, F.; Tsuchiya, H.; Tanaka, K.; Saeki, Y. Methods to measure ubiquitin chain length and linkage. Methods Enzymol. 2019, 618, 105-133. [CrossRef]

73. Enenkel, C.; Lehmann, A.; Kloetzel, P.M. Subcellular distribution of proteasomes implicates a major location of protein degradation in the nuclear envelope-ER network in yeast. EMBO J. 1998, 17, 6144-6154. [CrossRef]

74. Wilkinson, C.R.; Wallace, M.; Morphew, M.; Perry, P.; Allshire, R.; Javerzat, J.P.; McIntosh, J.R.; Gordon, C. Localization of the $26 \mathrm{~S}$ proteasome during mitosis and meiosis in fission yeast. EMBO J. 1998, 17, 6465-6476. [CrossRef]

75. Amsterdam, A.; Pitzer, F.; Baumeister, W. Changes in intracellular localization of proteasomes in immortalized ovarian granulosa cells during mitosis associated with a role in cell cycle control. Proc. Natl. Acad. Sci. USA 1993, 90, 99-103. [CrossRef]

76. Tsuchiya, H.; Arai, N.; Tanaka, K.; Saeki, Y. Cytoplasmic proteasomes are not indispensable for cell growth in Saccharomyces cerevisiae. Biochem. Biophys. Res. Commun. 2013, 436, 372-376. [CrossRef] [PubMed]

77. Shin, Y.; Brangwynne, C.P. Liquid phase condensation in cell physiology and disease. Science 2017, 357. [CrossRef] [PubMed] 
78. Sung, M.K.; Porras-Yakushi, T.R.; Reitsma, J.M.; Huber, F.M.; Sweredoski, M.J.; Hoelz, A.; Hess, S.; Deshaies, R.J. A conserved quality-control pathway that mediates degradation of unassembled ribosomal proteins. eLife 2016, 5. [CrossRef]

79. Nguyen, A.T.; Prado, M.A.; Schmidt, P.J.; Sendamarai, A.K.; Wilson-Grady, J.T.; Min, M.; Campagna, D.R.; Tian, G.; Shi, Y.; Dederer, V.; et al. UBE2O remodels the proteome during terminal erythroid differentiation. Science 2017, 357. [CrossRef] [PubMed]

80. Yanagitani, K.; Juszkiewicz, S.; Hegde, R.S. UBE2O is a quality control factor for orphans of multiprotein complexes. Science 2017, 357, 472-475. [CrossRef]

81. Jacobson, A.D.; MacFadden, A.; Wu, Z.; Peng, J.; Liu, C.W. Autoregulation of the $26 \mathrm{~S}$ proteasome by in situ ubiquitination. Mol. Biol. Cell 2014, 25, 1824-1835. [CrossRef]

82. Yoshizawa, T.; Ali, R.; Jiou, J.; Fung, H.Y.J.; Burke, K.A.; Kim, S.J.; Lin, Y.; Peeples, W.B.; Saltzberg, D.; Soniat, M.; et al. Nuclear Import Receptor Inhibits Phase Separation of FUS through Binding to Multiple Sites. Cell 2018, 173, 693-705.e622. [CrossRef] [PubMed]

83. Conicella, A.E.; Dignon, G.L.; Zerze, G.H.; Schmidt, H.B.; D’Ordine, A.M.; Kim, Y.C.; Rohatgi, R.; Ayala, Y.M.; Mittal, J.; Fawzi, N.L. TDP-43 alpha-helical structure tunes liquid-liquid phase separation and function. Proc. Natl. Acad. Sci. USA 2020, 117, 5883-5894. [CrossRef] [PubMed]

84. Li, P.; Banjade, S.; Cheng, H.C.; Kim, S.; Chen, B.; Guo, L.; Llaguno, M.; Hollingsworth, J.V.; King, D.S.; Banani, S.F.; et al. Phase transitions in the assembly of multivalent signalling proteins. Nature 2012, 483, 336-340. [CrossRef]

85. Raasi, S.; Orlov, I.; Fleming, K.G.; Pickart, C.M. Binding of polyubiquitin chains to ubiquitin-associated (UBA) domains of HHR23A. J. Mol. Biol. 2004, 341, 1367-1379. [CrossRef]

86. Dao, T.P.; Kolaitis, R.M.; Kim, H.J.; O’Donovan, K.; Martyniak, B.; Colicino, E.; Hehnly, H.; Taylor, J.P.; Castañeda, C.A. Ubiquitin Modulates Liquid-Liquid Phase Separation of UBQLN2 via Disruption of Multivalent Interactions. Mol. Cell 2018, 69, 965-978. [CrossRef] [PubMed]

87. Dao, T.P.; Martyniak, B.; Canning, A.J.; Lei, Y.; Colicino, E.G.; Cosgrove, M.S.; Hehnly, H.; Castañeda, C.A. ALS-Linked Mutations Affect UBQLN2 Oligomerization and Phase Separation in a Position- and Amino Acid-Dependent Manner. Structure 2019, 27, 937-951. [CrossRef] [PubMed]

88. Sun, D.; Wu, R.; Zheng, J.; Li, P.; Yu, L. Polyubiquitin chain-induced p62 phase separation drives autophagic cargo segregation. Cell Res. 2018, 28, 405-415. [CrossRef] [PubMed]

89. Laporte, D.; Salin, B.; Daignan-Fornier, B.; Sagot, I. Reversible cytoplasmic localization of the proteasome in quiescent yeast cells. J. Cell Biol. 2008, 181, 737-745. [CrossRef]

90. Kaganovich, D.; Kopito, R.; Frydman, J. Misfolded proteins partition between two distinct quality control compartments. Nature 2008, 454, 1088-1095. [CrossRef]

91. Janer, A.; Martin, E.; Muriel, M.P.; Latouche, M.; Fujigasaki, H.; Ruberg, M.; Brice, A.; Trottier, Y.; Sittler, A. PML clastosomes prevent nuclear accumulation of mutant ataxin-7 and other polyglutamine proteins. J. Cell Biol. 2006, 174, 65-76. [CrossRef] [PubMed]

92. Turakhiya, A.; Meyer, S.R.; Marincola, G.; Böhm, S.; Vanselow, J.T.; Schlosser, A.; Hofmann, K.; Buchberger, A. ZFAND1 Recruits p97 and the 26S Proteasome to Promote the Clearance of Arsenite-Induced Stress Granules. Mol. Cell 2018, 70, 906-919.e907. [CrossRef] [PubMed]

(C) 2020 by the authors. Licensee MDPI, Basel, Switzerland. This article is an open access article distributed under the terms and conditions of the Creative Commons Attribution (CC BY) license (http://creativecommons.org/licenses/by/4.0/). 